\title{
RESEARCH HIGHLIGHT Do striatal push/pull circuits hold the key to compulsive, relapsing heroin addiction?
}

\author{
A Research Highlight on: Chemogenetic modulation of accumbens direct or indirect pathways bidirectionally alters \\ reinstatement of heroin-seeking in high- but not low-risk rats, by O'Neal et al. (2019)
}

Morgan H. James $\mathbb{D}^{1,2}$ and Stephen V. Mahler $\mathbb{D}^{3}$

Neuropsychopharmacology (2020) 45:1243-1244; https://doi.org/10.1038/s41386-020-0615-1

Many people use opioid drugs medically and some abuse them recreationally, but only a few of these go on to develop diagnosable substance use disorders [1]. Thus, a major goal of addiction neuroscience should be to identify the biological mechanisms that predispose certain individuals to problematic drug use. Preclinical studies model this phenomenon by identifying subpopulations of laboratory animals - typically rodents - that exhibit addiction-like compulsive seeking approximating the selfdestructive, compulsive seeking observed in clinical populations. This approach offers a valuable platform to interrogate the neural mechanisms of addiction-like behavior, rather than the mechanisms of drug self-administration displayed by laboratory animals with few disincentives to pursuing addictive drugs [2].

Toward this end, O'Neal and colleagues [3] describe a novel approach for identifying rats with apparent high versus low vulnerability for heroin taking and seeking. The authors trained rats to self-administer heroin on an intermittent access (IntA) schedule, which results in repeated spikes in brain drug concentrations, and which potently promotes addiction-like endophenotypes with other abused drugs [4]. Next, they tested the rats for motivated responding for heroin on a progressive ratio schedule, as well as cue-induced reinstatement following extinction training, an established model of drug relapse. Mirroring a seminal prior report on cocaine addiction-like behavior in rats [5], the authors measured six putatively DSM-5 substance use disorder-relevant behavioral measures and computed an overall 'addiction severity' score for each individual rat. These included (1) heroin intake across IntA training; (2) consistency of responding during IntA training; (3) drug seeking during periods of signaled non-drug-availability throughout IntA training; (4) motivation for heroin, as indicated by break point on the progressive ratio test; (5) resistance to extinction training; and (6) 'relapse' vulnerability on the cue-induced reinstatement test. Using this approach, the authors identified subpopulations of 'vulnerable' and 'resilient' rats that tended to show clusters of addiction-like symptoms. Notably, although most rats were high on more than one index, these were not always the same across rats, potentially indicating subtypes of addiction-like behaviors even within this "vulnerable" group.

Next the authors sought to interrogate brain circuits that may function differently in "vulnerable" and "resilient" rats, testing here the relative roles of nucleus accumbens (NAc) efferent projections in addiction-like behaviors. Like dorsal striatum, NAc contains two interspersed populations of GABAergic medium spiny neurons that are differentiated according to their projections and other characteristics; direct pathway medium spiny neurons (dMSNs) that project directly to ventral tegmental area (VTA), and indirect pathway MSNs (iMSNs) that project to ventral pallidum (VP). These populations in more dorsal regions of striatum are often conceived of as playing facilitatory (dMSNs) or inhibitory (iMSNs) roles in performing actions. However, the functions of these opposing cell populations in NAc with respect to mediating opioid behaviors was previously untested.

Because the majority of dMSN vs. iMSN pathway mapping has been characterized in mouse, $\mathrm{O}^{\prime} \mathrm{Neal}$ and colleagues first tested whether these projections are segregated in rat. They achieved this by injecting two retrogradely transported $\mathrm{CTb}$ constructs with different fluorescent tags into VTA and VP and examining the expression of each tracer in NAc neurons. The large majority of neurons provided input to either VTA ( $50 \%)$ or VP (40-45\%) only, with only a small proportion of neurons $(5-10 \%)$ providing input to both structures. Likewise, when $\mathrm{O}^{\prime} \mathrm{Neal}$ et al. combined retrograde CAV2-Cre injections into VTA or VP with striatal injections of Credependent DREADD viruses, few infected neurons in either pathway appeared to project to the opposite pathway's target. Notably, this relatively strict anatomical segregation differs from recent results in reporter mice using other experimental methods [6], suggesting that most NAc neurons projecting to midbrain also provide synaptic input to VP. The nature of this apparent discrepancy should be pursued in future investigations. Regardless, O'Neal et al.'s data provide justification for using a retrograde transfection approach to selectively manipulate NAc dMSNs vs. iMSNs with DREADDs, to examine their role in heroin seeking in the vulnerable vs. resilient rats. Laudably, the authors confirmed the efficacy of both the excitatory and inhibitory DREADDs to alter neural activity with an amphetamine challenge. They showed that Fos in NAc was increased by activating dMSNs with hM3Dq, or by inactivating iMSNs with hM4Di-whereas Fos was decreased with the opposite manipulations in these cell types.

Next, separate groups of rats that were previously identified as addiction-vulnerable or resilient received viral injections to transfect dMSNs or iMSNs with either hM3Dq or hM4Di, allowing bidirectional manipulation of these populations during cue-induced heroin

\footnotetext{
${ }^{1}$ Brain Health Institute, Rutgers University, Piscataway, NJ, USA; ${ }^{2}$ Florey Institute of Neuroscience and Mental Health, University of Melbourne, Parkville, VIC, Australia and ${ }^{3}$ Department of Neurobiology \& Behavior, University of California Irvine, Irvine, CA, USA

Correspondence: Morgan H. James (morgan.james@rutgers.edu)
}

Received: 26 December 2019 Accepted: 30 December 2019

Published online: 23 January 2020 
reinstatement tests. In vulnerable rats, activation of dMSNs enhanced, and their inactivation suppressed, cued reinstatement. Manipulations of iMSNs in vulnerable rats had opposite effects, with activation and inhibition of these cells suppressing and enhancing reinstatement, respectively. Moreover, the magnitude of behavioral effects induced by DREADD manipulations was correlated with the rats' 'addiction severity scores', such that the greatest effects were observed in rats with higher scores. In contrast, equivalent modulation of neither dMSNs nor iMSNs affected cued reinstatement behavior in resilient rats. These data support the authors' interpretation that dMSNs promote, and iMSNs inhibit cue-induced reinstatement of heroin seeking selectively in the vulnerable rats. Moreover, when the authors performed the same DREADD manipulations during progressive ratio responding for heroin itself, manipulations of neither dMSNs nor iMSNs affected motivation to receive heroin, regardless of addiction vulnerability. This finding supports the notion that dissociable mechanisms control heroin selfadministration versus reinstatement of drug seeking [7].

Together, the findings of $\mathrm{O}^{\prime} \mathrm{Neal}$ and colleagues identify important new anatomical and functional data showing that segregated NAC circuits play distinct roles in cue-induced reinstatement of heroin seeking, especially in the more "vulnerable" rats. They also help contextualize our recent report that chemogenetic inhibition of VP neurons themselves (the target of NAc iMSNs examined here) has the most marked effects in the individual rats that most compulsively sought cocaine [8]. Such circuits as those reported here, which selectively mediate drug seeking in 'vulnerable' rats without similarly impacting'resilient' rats would be attractive targets for future clinical intervention to help people control their opiate use. Whether the striatal output pathways identified here fit this bill remains to be seen, but $\mathrm{O}^{\prime} \mathrm{Neal}$ et al. provide an important new lead for further research into the neural circuits underlying addiction to heroin-a vital public health concern worldwide at present.

\section{FUNDING AND DISCLOSURE}

This work was supported by financial support from the National Institute of Drug Abuse (K99DA045765 and P50DA044118). The authors have no other disclosures to report.

\section{ACKNOWLEDGEMENTS}

We would like to thank Dr. Yavin Shaham for his insightful comments on this manuscript.

\section{AUTHOR CONTRIBUTIONS}

MHJ and SVM drafted the manuscript, edited the manuscript and approved the final version of the manuscript.

\section{ADDITIONAL INFORMATION}

Publisher's note Springer Nature remains neutral with regard to jurisdictional claims in published maps and institutional affiliations.

\section{REFERENCES}

1. Shah A, Hayes CJ, Martin BC. Characteristics of initial prescription episodes and likelihood of long-term opioid use-United States, 2006-2015. Morbidity Mortal Wkly Rep. 2017;66:265-69.

2. Ahmed SH. The science of making drug-addicted animals. Neuroscience 2012;211:107-25.

3. O'Neal TJ, Nooney MN, Thien K, Ferguson SM. Chemogenetic modulation of accumbens direct or indirect pathways bidirectionally alters reinstatement of heroin-seeking in high- but not low-risk rats. Neuropsychopharmacology. 2019.

4. James MH, Stopper CM, Zimmer BA, Koll NE, Bowrey HE, Aston-Jones G Increased number and activity of a lateral subpopulation of hypothalamic orexin/hypocretin neurons underlies the expression of an addicted state in rats. Biol Psychiatry. 2019:85:925-35.

5. Deroche-Gamonet V, Belin D, Piazza PV. Evidence for addiction-like behavior in the rat. Science 2004;305:1014-7.

6. Kupchik YM, Brown RM, Heinsbroek JA, Lobo MK, Schwartz DJ, Kalivas PW. Coding the direct/indirect pathways by D1 and D2 receptors is not valid for accumbens projections. Nat Neurosci 2015;18:1230-2.

7. Shalev U, Grimm JW, Shaham Y. Neurobiology of relapse to heroin and cocaine seeking: a review. Pharmacol Rev 2002;54:1-42.

8. Farrell MR, Ruiz CM, Castillo E, Faget L, Khanbijian C, Liu S, et al. Ventral pallidum is essential for cocaine relapse after voluntary abstinence in rats. Neuropsychopharmacology. 2019;44:2174-85. 